\title{
The High Order Interaction Solutions Comprising Lump Solitons for the (2+1)-dimensional Caudrey-Dodd-Gibbon-Kotera-Sawada Equation
}

\author{
Jian-Hong Zhuang ${ }^{1}$, Yaqing $\mathrm{Liu}^{1}$, Juan-Juan $\mathrm{Wu}^{1}$, Ping Zhuang ${ }^{2}$, Xin $\mathrm{Chen}^{1}$, and \\ Xiao-Yong Wen ${ }^{3}$ \\ ${ }^{1}$ Beijing Information Science and Technology University \\ ${ }^{2}$ Liaoning Institute of Science and Technology \\ ${ }^{3}$ Beijing Information Science and Technology University,
}

September 29, 2020

\begin{abstract}
This paper deals with localized waves in the (2+1)-dimensional Caudrey-Dodd-Gibbon-Kotera-Sawada (CDGKS) equation in the incompressible fluid. Based on Hirota's bilinear method, N-soliton solutions related to CDGKS equation are constructed. For the case $\mathrm{N}=5$ and $\mathrm{N}=6$, the exact expression of multiple localized wave solutions comprising lump solitons are obtained by using the long wave limit method. A variety of interactions are illustrated analytically and graphically. The influence of parameters on propagation is analyzed and summarized. The results and phenomena obtained in this paper enrich the dynamic behavior of the evolution of nonlinear localized waves.
\end{abstract}

\section{Hosted file}

Manuscript.pdf available at https://authorea.com/users/362919/articles/483865-the-highorder-interaction-solutions-comprising-lump-solitons-for-the-2-1-dimensional-caudreydodd-gibbon-kotera-sawada-equation 
figures/fig1/fig1-eps-converted-to.pdf 
figures/fig2/fig2-eps-converted-to.pdf 
figures/fig3/fig3-eps-converted-to.pdf 
figures/fig4/fig4-eps-converted-to.pdf 
figures/fig5/fig5-eps-converted-to.pdf 
figures/fig6/fig6-eps-converted-to.pdf 
figures/fig7/fig7-eps-converted-to.pdf 
figures/fig8/fig8-eps-converted-to.pdf 
figures/fig9/fig9-eps-converted-to.pdf 